\title{
Pump Dispensing Plastic Container Closure
}

National Cancer Institute

\section{Source}

National Cancer Institute. Pump Dispensing Plastic Container Closure. NCI Thesaurus.

Code C96131.

Closure dispensing pumps are used to dispense product from containers. 